\title{
Maneuvering target tracking with improved interactive multiple model algorithm
}

\author{
Meiyu Zhu' ${ }^{1}$, Guanyu Chen ${ }^{2}$, Weicun Zhang ${ }^{{ }^{*}}$ \\ ${ }^{1}$ School of Automation and Electrical Engineering, University of Science and Technology Beijing, \\ 30 Xueyuan Road Haidian District, Beijing, China \\ E-mail:weicunzhang@ustb.edu.cn \\ www.ustb.edu.cn \\ ${ }^{2}$ University of Nottingham, $N G 72 R D, U K$ \\ E-mail:eexgc10@nottingham.ac.uk
}

\begin{abstract}
With the rapid development of artificial intelligence, maneuvering target tracking technology has become a major aspect of scientific and technological research with increased performance requirements. Maneuvering target tracking technology is widely used in military and civilian fields. This paper presents a new maneuvering target tracking framework, which adopts an improved interactive multiple model (IMM) with new weighting algorithm. Some target tracking simulations have been conducted based on MATLAB to verify the effectiveness of the proposed maneuvering target tracking approach.
\end{abstract}

Keywords: Maneuvering target tracking, interactive multiple model, weighting algorithm.

\section{Introduction}

In recent decades, with the rapid development of artificial intelligence, target tracking technology has become a major aspect of scientific and technological research. Maneuvering target tracking technology is widely used in military and civilian fields. For example, in air defense and air traffic control, reliable and accurate tracking of targets is always the main purpose of target tracking system design. However, with the rapid development of modern military weapons and the continuous advancement of aerospace technology, the maneuverability of various maneuvering targets has become more complicated, the motion state is more difficult to estimate, and the target maneuvering methods are complex and diversified, making it more and more difficult to track maneuvering targets. The original maneuvering target tracking technology can no longer meet the needs of modern maneuvering target tracking requirements.

The two key parts of maneuvering target tracking are the maneuvering target tracking model and the adaptive filtering algorithm. Considering that the motion model of

\footnotetext{
*Corresponding author: W. Zhang, weicunzhang@ustb.edu.cn

(C) The 2021 International Conference on Artificial Life and Robotics (ICAROB2021), January 21 to 24, 2021
} 
the target will also change when the target is maneuvered, any single model will produce larger errors. Also, the tracking process is often accompanied by external interference, system parameter jumps and other problems, these problems will cause the tracking performances to deteriorate. Kalman filtering or multiple model adaptive estimation (MMAE) or [1-6] method is a good idea to solve such kind of problem. This article will focus on weighted multiple model adaptive filtering with applications in maneuvering target tracking.

This article first introduces several commonly used maneuvering target tracking models, and analyzes their advantages, disadvantages, and scope of application through simulation, and proposes directions for improvement. Then introduce and study the filtering algorithm of target tracking, mainly including Kalman filtering algorithm, extended Kalman filtering algorithm, unscented Kalman filtering algorithm, and analysis and summary through simulation. Then this paper proposes a new weighting algorithm based on the traditional MMAE algorithm, and proves its convergence. The simulation proves that the new weighting algorithm has the characteristics of fast convergence speed and small calculation amount. Finally, the new weighting algorithm is combined with the interactive multi-model filtering algorithm to track the maneuvering target. The experimental results show that the new algorithm can track the target well, and compared with the traditional algorithm, the tracking speed is faster and the error is smaller.

\section{Interactive multiple model estimation}

Suppose target motion can be described by $r$ model, model set $M=\left\{M_{1}, M_{2}, \cdots, M_{r}\right\}$

$M j$ :

$X(k+1)=F_{j}(k) X(k)+G_{j}(k) w_{j}(k)$

$Z(k)=F_{j}(k) X(k)+v_{j}(k)$

$w_{j}(k)$ and $v_{j}(k)$ are independent Gaussian noises. The covariance matrixes of $w_{j}\left(k\right.$ and $v_{j}(k)$ are $R_{j}$ and $Q_{j}$, respectively. $u_{j}(k-1)$ represents the probability of model $M j$, the model transition probabilities can be described by

$$
P=\left[\begin{array}{ccc}
p_{11} & \cdots & p_{1 r} \\
\vdots & \ddots & \vdots \\
p_{r 1} & \cdots & p_{r r}
\end{array}\right]
$$

IMM filtering has the following 4 steps:

Step 1: Interaction

$$
\begin{aligned}
& u_{i \mid j}(k-1 \mid k-1)=\frac{p_{i j} u_{i}(k-1)}{c_{j}} \\
& c_{j}=\sum_{i=1}^{r} p_{i j} u_{i}(k-1) \\
& \hat{X}_{0 j}(k-1 \mid k-1)=\sum_{i=1}^{r} \hat{X}_{i}(k-1 \mid k-1) u_{i \mid j}(k- \\
& 1 \mid k-1) \\
& \quad P_{0 j}(k-1 \mid k-1)=\sum_{i=1}^{r}\left\{P_{i}(k-1 \mid k-1)+\left[\hat{X}_{i}(k-\right.\right. \\
& \left.1 \mid k-1)-\hat{X}_{0 i}(k-1 \mid k-1)\right] \\
& \left.\left.\left[\widehat{X}_{i}(k-1 \mid k-1)-\hat{X}_{0 i}(k-1 \mid k-1)\right]^{T}\right]\right\} u_{i \mid j}(k- \\
& 1 \mid k-1)
\end{aligned}
$$

\section{Step 2: Filtering}

$$
\begin{aligned}
& \hat{X}_{j}(k \mid k-1)=F_{j}(k-1) \hat{X}_{0 j}(k-1 \mid k-1) \\
& P_{j}(k \mid k-1)=F_{j}(k-1) P_{0 j}(k-1 \mid k-1) F_{j}^{T}(k-1)+ \\
& \Gamma_{j}(k-1) Q_{j}(k-1) \Gamma_{j}^{T}(k-1) \\
& \varepsilon_{j}=Z(k)-H_{j} \hat{X}_{j}(k-1 \mid k-1) \\
& S_{j}(k)=H_{j}(k) P_{j}(k \mid k-1) H_{j}^{T}(k)+R_{j}(k) \\
& K_{j}(k)=P_{j}(k \mid k-1) H_{j}(k) S_{j}^{-1}(k) \\
& \hat{X}_{j}(k \mid k)=\hat{X}_{j}(k \mid k-1)+K_{j}(k) \varepsilon_{j}(k) \\
& P_{j}(k \mid k)=\left[I-K_{j}(k) H_{j}(k)\right] P_{j}(k \mid k-1)
\end{aligned}
$$

Step 3: Model probability updates

$$
\begin{aligned}
& l_{j}(0)=\frac{1}{m} ; u_{j}(0)=l_{j}(0) \\
& l_{j}^{\prime}(k)=1+\frac{1}{r} \sum_{q=1}^{r}\left\|\varepsilon_{j}(q)\right\|^{2} \\
& l_{\text {min }}^{\prime}=\min _{i}\left\{l_{j}^{\prime}(k)\right\} \\
& l_{j}(k)=\frac{l_{\min }^{\prime}}{l_{j}^{\prime}(k)} l_{j}(k-1) \\
& u_{j}(k)=\frac{1}{c} l_{j}(k) c_{j} \\
& c=\sum_{j=1}^{r} l_{j}(k) c_{j}
\end{aligned}
$$


Step 4: Combination

$$
\begin{aligned}
& \hat{X}(k \mid k)=\sum_{j=1}^{r} \hat{X}_{j}(k \mid k) u_{j}(k) \\
& P(k \mid k)=\sum_{j=1}^{r}\left\{P_{j}(k \mid k)+\left[\hat{X}_{j}(k \mid k)-\right.\right. \\
& \left.\left.\hat{X}(k \mid k)]\left[\hat{X}_{j}(k \mid k)-\hat{X}(k \mid k)\right]^{T}\right]\right\} u_{j}(k)
\end{aligned}
$$

\section{Simulation results}

To verify the effectiveness of the proposed maneuvering target tracking based on improved IMM algorithm, many simulations have been conducted with MATLAB platform. Simulation results are satisfactory, details are omitted here due to space limit, just to illustrate some pictures of simulation results. Figure 1 shows plane tracking performance comparisons among Current Statistical (CS) model, IMM algorithm, and improved IMM algorithm; Figure 2 and Figure 3 show tracking performance comparisons in X-direction and Ydirection; Figure 4 shows the weights values of IMM algorithm; Figure 5 shows the weights values of improved IMM algorithm.

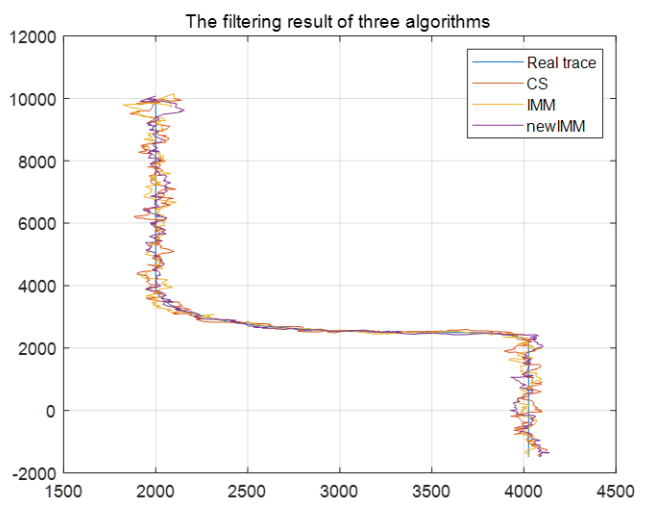

Figure 1 The filtering results of three algoritms

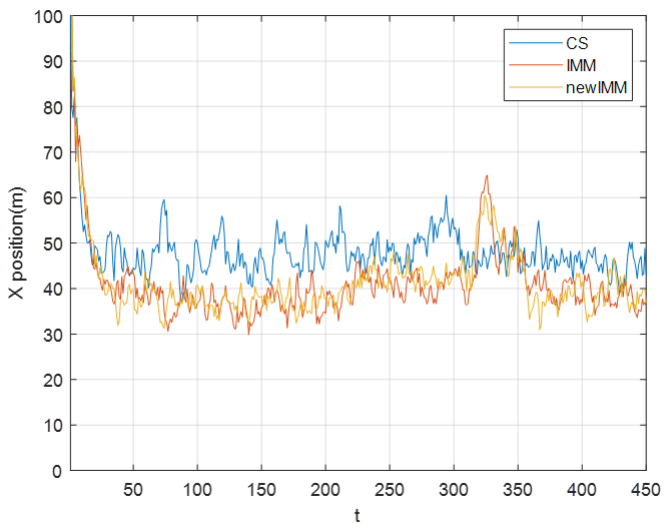

Figure 2 Positions: $\mathrm{X}$ direction

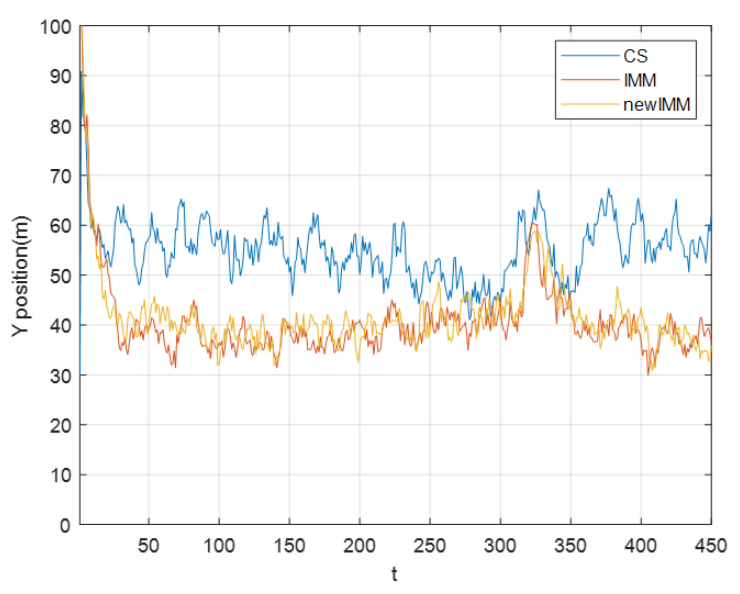

Figure 3 Positions: Y direction

Figure 4 Weight values of traditional IMM algorithm

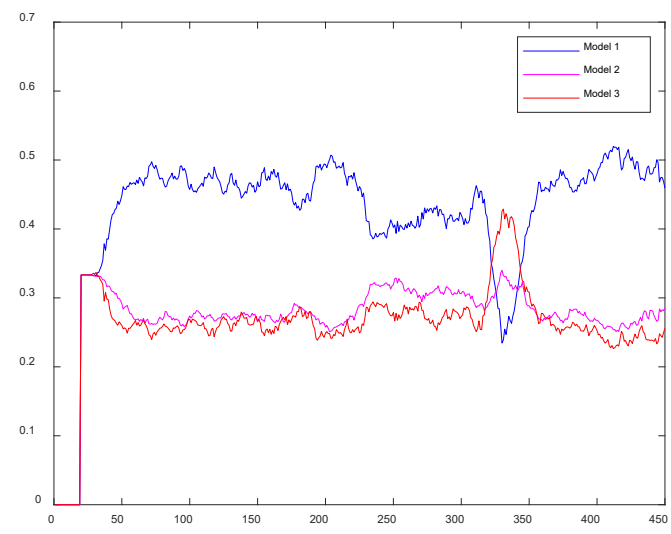

(C) The 2021 International Conference on Artificial Life and Robotics (ICAROB2021), January 21 to 24, 2021n 


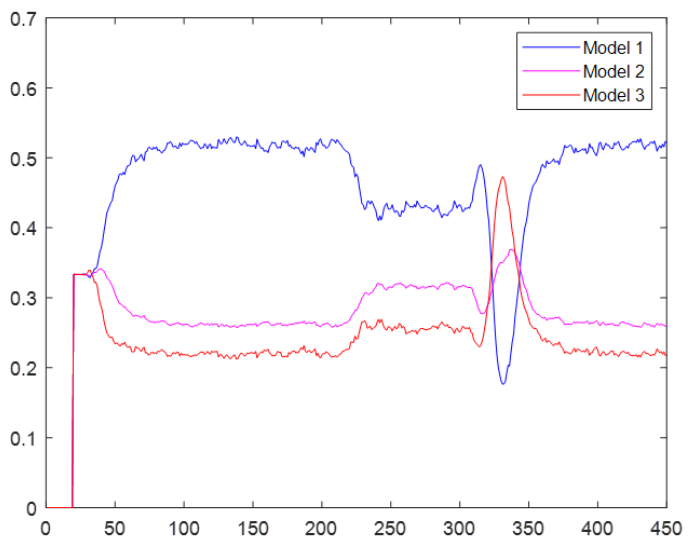

Figure 5 Weight values of improved IMM algorithm

\section{Conclusion and future work}

This paper presented an improved IMM maneuvering target tracking approach. Simulation results verified the effectiveness of the proposed method including new weighting algorithm. In the future research work, we will focus on model set selection with prescribed performance requirements.

\section{Acknowledgements}

This work was partly supported by National Natural Science Foundation of China (No. 61520106010; 61741302).

\section{References}

1. D. T. Magill, Optimal adaptive estimation of sampled stochastic processes, IEEE Trans. Automat. Contr., 1965; 10: $434-439$

2. D. G. Lainiotis, Partitioning: A unifying framework for adaptive systems-I: Estimation; II: Control, Proc. IEEE, 1976; 64:1126-1143 and 1182-1197

3. R. E. Kalman, "A new approach to linear filtering and prediction problems," Journal of Basic Engineering, vol. 82, no. 1, pp. 35-45, 1960.

4. P. Zarchan and H. Musoff, Fundamentals of Kalman Filtering: a Practical Approach, American Institute of Aeronautics and Astronautics, 4th edition, 2015.

5. X.-R. Li and Y. Bar-Shalom, "Multiple-model estimation with variable structure," IEEE Transactions on Automatic Control, vol. 41, no. 4, pp. 478-493, 1996.

6. W. Zhang, S. Wang, and Y. Zhang, Multiple-model adaptive estimation with a new weighting algorithm, Complexity, 2018:1-11,2018 